\title{
Status and Progress of Selective Laser Melting Forming Technology
}

\author{
Shuai Chen ${ }^{\text {a }}$, Fenghe Tao, Changzhi Jia and Jianchun Yang \\ Shijiazhuang Mechanical Engineering College, Shijiazhuang 050003, China \\ achenshuai680707@163.com
}

Keywords: rapid prototyping; SLM; major problems; development trend.

\begin{abstract}
Selective Laser Melting (SLM) was a novel method of Rapid Prototyping (RP). The benefits of SLM: simple process, good surface roughness, high-precision molding size, density of almost $100 \%$.The basic principles of the technique, the major problems and present situation in China and abroad were described and introduced. And discuss the development trend of the SLM technology.
\end{abstract}

\section{Introduction}

SLM technique was a novel method of Rapid Prototyping technology, appeared in the 1990s. It combines CAD/CAM, CNC, optics and material science. SLM technology was developed on the base of SLS. The technology fabricated metal parts direct using metal powder. SLM possessed the convenience of the SLS. Conmpare to the SLS technology, in the process of fabrication, SLM could get the metal parts with a metallurgical bond, compact structure and very high precision. This technology could avoid the post-treatment process of low melting point metal infiltration and this is the huge advantage in the process of metal parts fabrication.

SLM technique uses all kinds of pure metal powder or alloy metal powder for processing the raw material, and it uses the small and medium power laser melting the selective metal powder. After melting the powder, nonequilibrium state-supersaturated solid solution and finer microstructure were obtained by combining with Rapid cooling solidification technology [1].

The density of modeling part is almost $100 \%$, and the mechanical properties are equivalent to forging parts. SLM technique has advantages such as simple process, wide range of forming materials, and it can make complex metal parts that traditional methods can't. SLM technique has become the most promising technology in the Rapid Prototyping technology and it gains extensive attention by experts both in China and abroad.

Although SLM technology has the competitive advantage in the materials selection, design free degree and research cost and so on, the strict requirements to the equipment and the unstability of key technology confine the application and extension of SLM.

Metal parts could be directly manufactured with the selective melting technology. They are applied to biomedical field, aerospace field,etc. There are wide gaps in the SLM technologe home, compared with abroad. In the SLM processes, some problems, such as balling effect, crack, curl, distortion, delamination and low processing efficient, might occur.

\section{The basic principles of SLM}

SLM technology was a kind of advanced laser material manufacturing technology based on the basic principles of rapid prototyping technique.

The 3D model of element was sliced by specialized software. After getting the contour data of each section, it uses high-energy laser beam to melt the metal powder selectively according to the contour data. Spreading powder and melting solidification accumulation layer-by-layer to create the 3D entity elements. After slicing the 3D model of element and importing the data into the modeling equipment, the horizontal chain puts a thin layer of metal powder on the substrate evenly.

The high energy laser beam melts the powder on the substrate selectively according to the 3D model data of the current layer. After forming the shape of the current layer, the horizontal chain has 
putted another thin layer of metal powder on the substrate evenly. The high energy laser beam melts the powder on the substrate selectively according to the 3D model data of the next layer. Manufacturing the element by the cycling way until the whole parts completed [2].

\section{Major problems}

The research on the SLM now is focused on the fabrication process experiments, application fields' exploration and hardware development. Many problems have to be solved on hardware structure, fabrication process optimization and forming mechanism.

We need to address the problems such as the materials is expensive, the equipment is expensive and the complicated operations and other issues. Operating working device requires professionals to complete, and operators need to conduct in-depth understanding of the technical principles. Equipment maintenance costs more expensive, including the protection of gas, molding materials and so on. There are still some problems in the current development of domestic SLM technology. The stability of the domestic parts can't reach the level of foreign, currently only making some small size work pieces.

Metal powders currently used mostly for special powder, the less common and more expensive. Current conversion rate of the laser power is low; increasing conversion rate will greatly enhance the productivity of parts. Currently, most systems use open-loop control, there are defects in the metal parts to ensure dimensional accuracy. How to optimize the scan path of laser is a major work, it is possible to produce the deformation and stress of parts small enough.

In the processing of a complicated physical chemistry metallurgy, due to metal powders melting quickly, melting pond exiting short, large temperature gradient and stress caused by temperature gradient, and large surface tension of melting powders, there exits three internationalism problems which are warp, crack and balling phenomenon. In order to solve these problems, we need to bring up corresponding investigation schemes.

\section{Present situations}

At present, the United States, Europe and other developed countries take the lead position in the powder raw materials preparation, forming equipment, software and technology of SLM. EOS GmbH in Germany developed a novel forming equipment, it uses Yb fiber laser with high quality beam source. And it can focus the beam spot diameter to $0.1 \sim 0.5 \mathrm{~mm}$, increase the laser scanning speed and reduce the forming time. Its mechanical properties are equivalent to forging parts.

In recent years, Britain, Germany, France and other developed countries successively developed the forming equipment for commercialization using high-temperature alloy, AlSi10Mg, Ti6Al4V and other alloy. And they have applied the basic research for application. At present, the largest processing volume can reach $300 \mathrm{~mm} * 300 \mathrm{~mm} * 250 \mathrm{~mm}$ by using the forming equipment for commercialization [3].

In 2012, Eads shows the titanium alloy parts using SLM technique and they replace the cast steel hinge bracket in airbus A320 with the parts. It can optimize the location of the load, save the weight of $10 \mathrm{~kg}$ one suite, reduce the energy and mission in the process of production, operation and the end of the recycling [4].

NASA made a micro jet of the rocket engine using SLM technique, the length is $15.62 \mathrm{~cm}$. The same kind of the jet is composed of 115 parts in the previous test, but this new micro jet is composed of 2 parts. The cost of the jet reduces by more than $70 \%$, and the technique greatly shortens the development time. At the same time, the test shows that the micro jet works properly.

General Electric Corporation made a fuel nozzle using to its Leap engine. The new part using SLM technique replaced the traditional nozzle which is composed of 20 parts. And the new part reduces $25 \%$ in weight. 
Honeywell Corporation made the heat exchanger and metallic stent. United Technologies Corporation made the compressor blade in the jet engine and set up the SLM research center in the University of Connecticut [5].

In recent years, domestic related research institutions pay more attention to SLM technique. Now in our country, we have made significant progress in forming equipment, manufacturing technique, process control, process stability. In general, the hardware system, processing property, and the mechanical property of modeling part can reach or approach advanced international standards [6].

BAMTRI developed the SLM prototyping platform and had carried out research in the conventional manufacture methods using titanium alloy such as TC4, TC11, TB6, etc. They solved many technical difficult problems such as balling effect, distortion, defects control, active control on structure and property.

They master the SLM conventional manufacture methods, and the mechanical properties are equivalent to forging parts. Platinum Force Corporation has conducted the widespread thorough research to the technology of forming equipment, forming technology, active control on structure and property, temperature profiles, stress field and feedback control. They have realized the manufacture of complex shape parts using Nickel-based alloy, stainless steel, titanium alloy and other metal materials. In addition, Huazhong University and other units carried out the typically work on the SLM forming mechanism and techniques [7].

\section{Development trend of SLM}

The SLM forming parts applied greatly, such as the tool and die in the mechanical field, biomedical implant parts or replacement parts in the biomedical field, heat dissipation in the electronic field, light weight structure in the aerospace field, and functionally gradient composites material parts [8].

The more application in the field of aerospace is the typical varieties of small-scale production process. Especially in the development phase, the SLM technology has the incomparable advantage. The machining of some complex work pieces is not only a waste of time but also a serious waste of material. Some complex structure even can't make. Casting can solve the problem of complex structure and improve material utilization, but special materials such as titanium and nickel casting process is very complex. And product performance is difficult to control. Forging parts can effectively improve performance, but it needs expensive precision mold and large special equipment, and the manufacturing cost is very high. The SLM technology can easily and quickly create these complex work pieces. In the product development, it can shorten the processing time and save a lot of the development costs [9].

\section{Summary}

In general, the SLM technology represents the development direction in the field of rapid prototyping. Using this technology can directly form metallic dense-component with high complex structure, high dimensional accuracy and high surface quality. And it can reduce the manufacturing process of metal parts providing more efficient approach for the design and manufacture. So it can accelerate the response speed of product market and update the design concept and production cycle. SLM technology will be better and faster development in the future[10].

At present, the developed countries in terms of the technology of powder preparation, molding equipment, software production, etc. are in a leading position. China is still in catch-up phase of rapid development, we need to strive to catch up, formed a complete industrial chain. In order to make SLM technology to produce metal parts well, we need to improve part quality and reduce the difficulty of the production process, reduce production costs[11]. Only in this way, SLM technology can be more widely applied and play a better role in the national economy. 


\section{References}

[1] Liu Weijun. Rapid Prototyping Technology and Its Application [M]. Beijing: China Machine Press, 2005: 80-200.

[2] Wu Weihua, Yang Yongqiang, Wang Di. Research on variable density rapid manufacturing process based on selective laser melting technology [J]. Chinese J.Lasers, 2010, 37(7): 1879-1884.

[3] Huang Weidong, Li Yanmin, Feng Lipin. Laser solid forming of metal powder materials [J]. Material Engineering, 2002(3):40-43.

[4] Badrossamay M, Childs T H C. Further studies in selective laser melting of stainless and tool steel powders [J].International Journal of Machine Tools \& Mnufacture, 2007, 47:779-784.

[5] Buchbinder D, Schleifenbaum H, Heidrich S,et al. High powder selective laser melting of aluminum parts[J]. Physics Procedia, 2011, 12: 271-278.

[6] Amato KN, Gaytan SM, Murr L E, etal. Microstructures and mechanical behavior of Inconel 718 fabricated by selective laser melting [J]. ActaMaterialia, 2012, 60(5):2229-2239.

[7] EOS GmbH. the Product Instruction of EOSINT M270. Germany: EOS GmbH, 2005: 8-21.

[8] Lu L,Fuh J Y H, Chen Z D,et al. In situ formation of TiC composite using selective laser melting [J].Materials Characterization, 2011, 62 (5):488-495.

[9] Chu C, Graf G, Rosen D W. Design for additive manufacturing of cellular structures [J]. Computer-Aided Design and Applications, 2008, 5(5):686-696.

[10] Osakada K, Shiomi M. Flexible manufacturing of metallic Products by selective laser melting of Powder [J]. International Journal of Machine Tools \& Manufacture, 2006, 46 (11):1188-1193.

[11] Over C, Meiners W W, issenbach K. Selective laser melting: a new approach for the direct manufacturing of metal parts and tools [A]. 1st International Conference on Laser Assisted Net Shape Engineering[C]. Germany: Frankfurt, 2001:22-35. 